\title{
Characteristics of fiber-optic magnetic-field sensors employing metallic glasses
}

\author{
K. P. Koo and G. H. Sigel, Jr. \\ Naval Research Laboratory, Washington, D.C. 20375
}

Received March 1, 1982

\begin{abstract}
A variety of extremely high-sensitivity, room-temperature magnetic-field sensors have been fabricated and tested using single-mode fibers in conjunction with highly magnetostrictive metallic glasses. Minimum detectable fields of $5 \times 10^{-9} \mathrm{Oe} / \mathrm{m}$ fiber are reported. The typical characteristics of the fiber magnetometers are summarized.
\end{abstract}

It has been shown by Dandridge et al. ${ }^{1}$ that nickel magnetostrictive jackets and coatings on single-mode fibers deployed in a Mach-Zehnder interferometer can be utilized in the laboratory to measure magnetic fields in the range of $10^{-6} \mathrm{Oe}$ for sensor lengths of the order of $1 \mathrm{~m}$. Subsequently, both phase modulation ${ }^{2}$ and magnetic sensing ${ }^{3}$ using metallic glasses in conjunction with optical fibers have been reported. This Letter describes in detail the use of commercial metallic-glass magnetostrictive materials on fibers for very-low-field magnetic-sensor applications. This approach has resulted in a dramatic improvement of magnetic-field sensitivity with potentially reduced magnetic noise and low bias fields while both simple fiber-sensor configurations and the excellent linearity of response versus applied magnetic field have been maintained. The data reported here represent by far the highest sensitivities achieved to date to our knowledge for fiber magnetometers and would appear to make these devices serious candidates for a variety of magnetic-sensor applications.

The experimental details of the all-fiber interferometer employed to measure small phase shifts have been described. ${ }^{4}$ The basic approach for magnetic-field detection is to bond a fiber in one arm of the interferometer to a magnetostrictive material that changes linear dimension in an applied magnetic field. Figures 1(a) and 1(b) show schematically two of the specific configurations utilized in the laboratory to evaluate metallic-glass fiber magnetometers. In Fig. 1(a), two pairs of the Helmholtz coils are used to generate ac and dc magnetic fields, respectively, for a fiber sensor consisting of a bare single-mode (o.d. $\approx 80 \mu \mathrm{m}$ ) fiber bonded to a metallic-glass strip. Commercially available metallic glasses, such as the Allied Chemical \#2605 SC, \#2605 CO, and \#2826 MB alloys, have been found to provide excellent performance as magnetostrictive materials in the fiber magnetometers. Figure 1(b) shows an alternative configuration in which the metallic glass is wrapped around a current-carrying copper cylinder. Local magnetic fields are generated by electrical currents passing through the cylinder. The fiber has been bonded to the metallic glass to provide a magnetically active path length of the order of $1 \mathrm{~m}$. The data provided here are typical of the best commercially available metallic glasses, but the magnetomechanical response of these materials can be further improved by thermal annealing in constant magnetic fields under reducing conditions. ${ }^{5,6}$

In order to characterize the performance of the fiber magnetic sensors in the laboratory, it is essential to

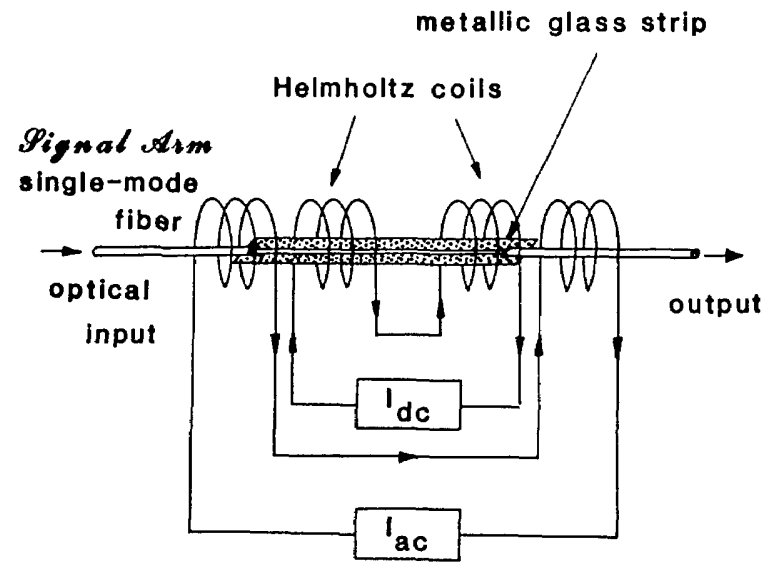

(a)

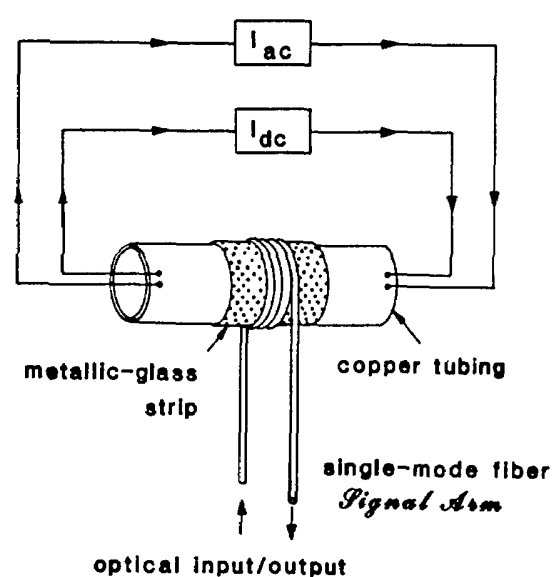

(b)

Fig. 1. Schematics of the magnetic-sensor configurations: (a) strip geometry, (b) tubular geometry. 


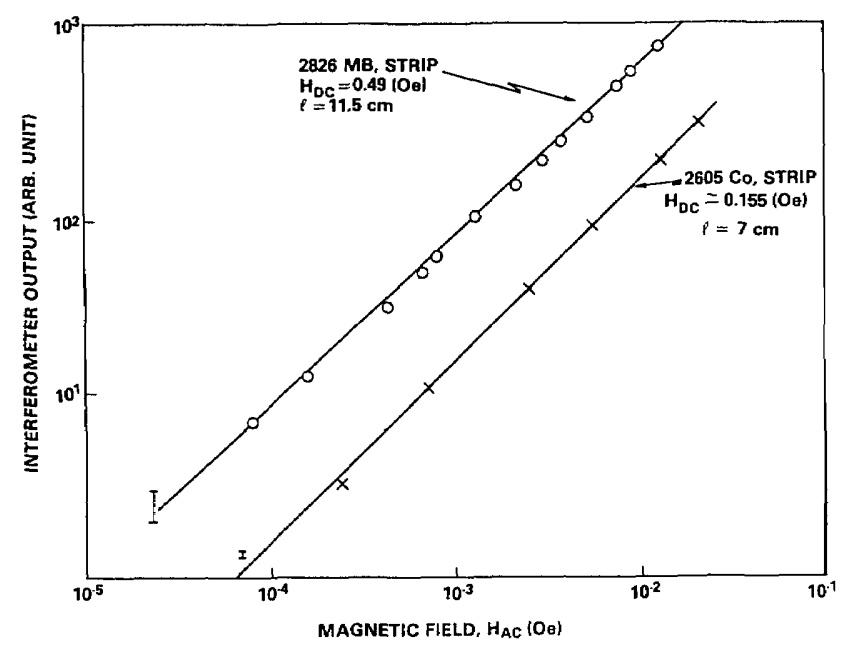

Fig. 2. Linearity of interferometer responses versus magnetic fields.

measure a variety of parameters, including linearity of response to applied magnetic field, the relative sensitivity as a function of applied field frequency, the effects of dc magnetic-bias field on the device output, orientation response, and the impact of magnetic noise on device sensitivity. Figure 2 shows the excellent response linearities of the \#2605 CO and \#2826 MB metallic glasses as a function of applied ac magnetic field at the frequency of $1 \mathrm{kHz}$. The dynamic range of the linear response of the fiber-optic magnetic sensors is limited only at high magnetic fields. This limitation occurs because a standard phase-modulated signal will follow a Bessel-function relation as a function of signal strength. To date, all fiber samples configured with either bonded metallic-glass strips or evaporated coatings have exhibited excellent linearity of response versus applied magnetic field.

Another important parameter of interest in assessing sensor performance is the magnetic-bias-field dependence of the magnetostriction. Typically the induced oscillation of a magnetostrictively jacketed fiber in an ac magnetic field will vary in magnitude as a function of the applied or ambient constant magnetic field. Peaking the response of the device therefore requires the identification of the optimum bias conditions to achieve maximum metallic-glass magnetostriction. Figure 3 shows the measured bias-field dependence for. fiber sensors employing metallic glasses \#2605 CO, \#2605 SC, and \#2826 MB at a frequency of $1 \mathrm{kHz}$. No significant changes have been observed for the optimal bias fields in a frequency range up to a few kilohertz for each sample that was studied. A broad maximum centered near a 5-Oe bias field was observed for the \#2605 CO and \#2826 MB strip-form devices [Fig. $1(a)$, but for the \#2605 SC material in a tubular geometry [Fig. 1(b)] a relatively sharp bias characteristic occurs in the vicinity of a $0.9-\mathrm{Oe}$ bias field. Bias-field behavior is related to the magnitude and distribution of the magnetic moments in the metallic glasses and can be readily affected by field annealing of samples before measurement.
Whereas the dc bias field is maintained at a fixed value representing the optimum magnetic biasing of the magnetostrictive device, the frequency of a constantmagnitude ac test signal can be varied to measure the frequency response of the fiber-optic sensor. Some typical frequency-response data are shown in Fig. 4 for both tubular and strip-line metallic-glass sensors. The geometry of the sensors can dramatically affect the measured frequency characteristic. Sharp resonances are often observed in the strip configurations for strip sizes of the order of $1.25 \mathrm{~cm} \times 15 \mathrm{~cm} \times 50 \mu \mathrm{m}$. Any loading or stressing of the fiber sensor will also affect the amplitude and the phase of the optical signal. Because of this sensitivity to applied force, it is essential to utilize bonding agents that exhibit low coefficients of expansion. The smooth frequency response of the metallic glasses deployed in a tubular configuration appears to be related to both geometric and loading effects. Since all data presented here are for as-received metallic

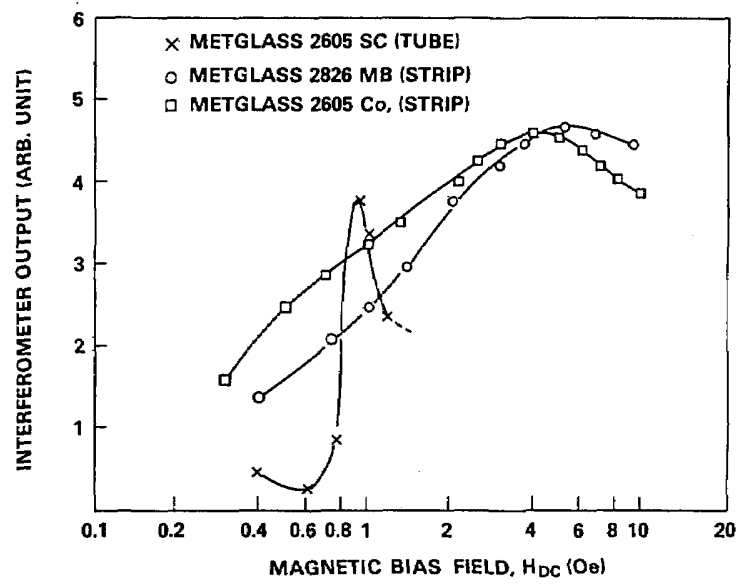

Fig. 3. Interferometer responses versus magnetic-bias fields.

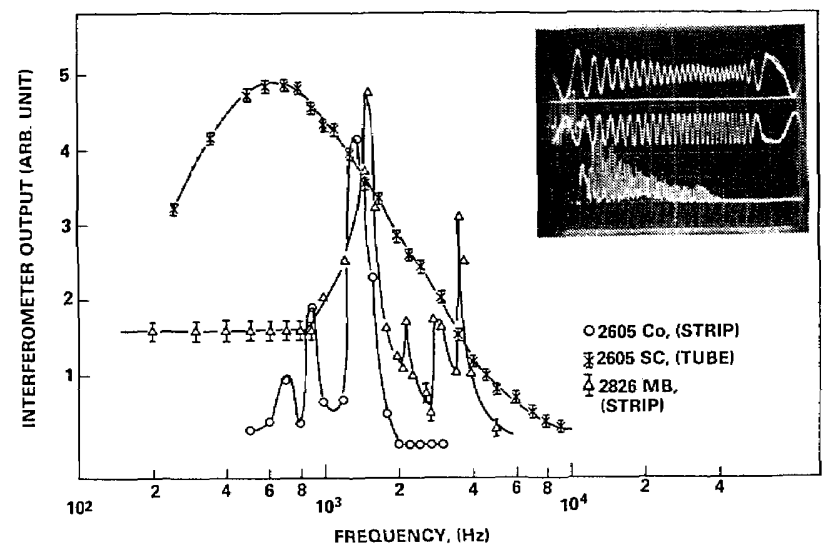

Fig. 4. Frequency responses of three magnetic sensors using three different types of metallic glass and two different sensor geometries. The insert shows an oscilloscope trace of the frequency response for metallic glass \#2605 SC in tubular configuration: upper trace is the interferometer response, middle trace is the sweep-frequency drive $(0.5 \mathrm{~Hz}$ to $5 \mathrm{~Hz})$ to the Helmholtz coil, and lower trace is the spectrum of the interferometer output. 
Table 1. Sensitivities of Metallic-Glass Fiber-Optic Magnetic Sensors ${ }^{a}$

\begin{tabular}{|c|c|c|}
\hline $\begin{array}{c}\text { Sensor } \\
\text { Configuration } \\
\left(\text { Material Type }^{b}\right) \\
\end{array}$ & $\begin{array}{c}\text { Magnetic-Bias } \\
\text { Field } \\
H_{\mathrm{dc}}(\mathrm{Oe}) \\
\end{array}$ & $\begin{array}{c}\text { Minimum } \\
\text { Detectable Field } \\
\text { (Oe/m of Fiber) }\end{array}$ \\
\hline $\begin{array}{l}\text { Strip line, } \| \text { to axis } \\
\quad(\# 2605 \mathrm{CO})\end{array}$ & 0 & $1.5 \times 10^{-8}$ \\
\hline $\begin{array}{l}\text { Strip line, } \| \text { to axis } \\
\quad(\# 2605 \mathrm{CO})\end{array}$ & 6 & $2.3 \times 10^{-8}$ \\
\hline $\begin{array}{l}\text { Strip line, } \frac{\perp}{1} \text { to axis } \\
\quad(\# 2605 \mathrm{CO})\end{array}$ & 28 & $1 \times 10^{-7}$ \\
\hline $\begin{array}{l}\text { Cylinder, It to axis } \\
(\# 2605 \text { SC) }\end{array}$ & 0 & $2 \times 10^{-6}$ \\
\hline $\begin{array}{l}\text { Cylinder, } \| \text { to axis } \\
(\# 2605 \text { SC) }\end{array}$ & 1.0 & $5 \times 10^{-9}$ \\
\hline $\begin{array}{l}\text { Strip line, } \| \text { to axis } \\
\quad(\# 2826 \mathrm{MB})\end{array}$ & 3.5 & $6 \times 10^{-9}$ \\
\hline
\end{tabular}

${ }^{a}$ Data measured for $1-\mathrm{kHz}$ applied ac magnetic field.

${ }^{b}$ Metallic-glass numbers are designations of Allied Chemical Corporation, Morristown, N.J.

glasses that have not been subjected to field anneals, differing residual strains in various samples will cause substantial variations in device performance. Efforts are under way both to interpret the observed frequency-response variations observed in the metallicglass optical-fiber sensors and to eliminate them by proper sample fabrication and anneal schedules.

Perhaps the parameter of greatest interest in the evaluation for potential device application is the minimum detectable magnetic field that can be measured. Sensitivity data are given in Table 1 for the three metallic glasses measured in this study. Results are included for both strip-line and tubular configurations and a variety of bias-field conditions. The data correspond to the best sensitivities obtained to date among a number of samples tested in each category. These lower limits on detectable magnetic fields are established by the resolution of the optical interferometer, which is capable of detecting phase shifts as small as a microradian. It is assumed that the magnetostriction versus applied magnetic-field curve remains linear over the region of interest. Table 1 also shows comparisons of data taken on fibers bonded perpendicular to the ribbon axis (hard magnetic direction) with those taken for fibers oriented parallel to the ribbon. Typical results showed a 20-30-fold reduction in sensitivity in the hard direction. Minimum detectable fields in the range of $5 \times 10^{-9} \mathrm{Oe} / \mathrm{m}$ of fiber were obtained on a variety of samples subjected to $1-\mathrm{kHz}$ magnetic signals. This corresponds to a measurable phase shift of $10^{-6} \mathrm{rad}$ with a signal-processing bandwidth of $1 \mathrm{~Hz}$. This is an excellent response for a room-temperature device.

In conclusion, high-sensitivity magnetic-field sensors employing metallic glasses have been fabricated and shown to be capable of detecting minimum magnetic fields of the order of $5 \times 10^{-9} \mathrm{Oe} / \mathrm{m}$ fiber. Improved devices with even higher sensitivity and more-uniform frequency response are currently being investigated. However, the excellent sensitivities already measured in this work would appear to make fiber magnetometer devices serious candidates for a variety of magneticsensor applications.

\section{References}

1. A. Dandridge, A. B. Tveten, G. H. Sigel, E. J. West, and T. G. Giallorenzi, "Optical fiber magnetic field sensors," Electron. Lett. 16, 408-409 (1980).

2. F. R. Trowbridge and R. L. Phillips, "Metallic-glass fiber-optic phase modulators," Opt. Lett. 6, 636-639 (1981).

3. H. F. Taylor, T. G. Giallorenzi, and G. H. Sigel, "Fiber optic sensor," Proc. IEE 201, 99-101 (1981).

4. D. A. Jackson, A. Dandridge, and S. K. Sheem, "Measurement of small phase shifts using a single-mode optical interferometer," Opt. Lett. 5, 139-141 (1980).

5. C. Modzelewski, H. T. Savage, L. T. Kabacoff, and A. G. Clark, "Magnetomechanical coupling and permeability in transversely annealed metglass 2605 alloys," IEEE Trans. Magn. M-17, 2837-2839 (1981).

6. M. A. Mitchell, A. Clark, H. T. Savage, and R. J. Abbundi, " $\Delta E$ effect and magnetomechanical coupling Factor in $\mathrm{Fe}_{80} \mathrm{~B}_{20}$ and $\mathrm{Fe}_{78} \mathrm{Si}_{70} \mathrm{~B}_{12}$ glassy ribbons," IEEE Trans. Magn. M-14, 1169-1171 (1978). 